\title{
Women in the 2019 hepatitis C cascade of care: findings from the British Columbia Hepatitis Testers cohort study
}

\author{
Margo E. Pearce ${ }^{1,2^{*}}$, Sofia R. Bartlett ${ }^{1,3}$, Amanda Yu' ${ }^{1}$, Jess Lamb ${ }^{4}$, Cheryl Reitz ${ }^{5,6}$, Stanley Wong ${ }^{1}$, Maria Alvarez ${ }^{1}$, \\ Mawuena Binka ${ }^{1}$, Héctor Velásquez Garcia ${ }^{1}$, Dahn Jeong ${ }^{1,2}$, Emilia Clementi ${ }^{1,2}$, Prince Adu ${ }^{1,2}$, Hasina Samji ${ }^{1}$, \\ Jason Wong ${ }^{1,2}$, Jane Buxton 1,2, Eric Yoshida ${ }^{7,8}$, Chelsea Elwood ${ }^{9,10}$, Laura Sauve ${ }^{10,11,12}$, Neora Pick ${ }^{10,13}$, \\ Mel Krajden ${ }^{1,3}$ and Naveed Z. Janjua ${ }^{1,2}$
}

\begin{abstract}
Background: Women living with hepatitis $\mathrm{C}$ virus $(\mathrm{HCV})$ are rarely addressed in research and may be overrepresented within key populations requiring additional support to access HCV care and treatment. We constructed the HCV care cascade among people diagnosed with HCV in British Columbia, Canada, as of 2019 to compare progress in care and treatment and to assess sex/gender gaps in HCV treatment access.

Methods: The BC Hepatitis Testers Cohort includes 1.7 million people who tested for HCV, HIV, reported cases of hepatitis B, and active tuberculosis in BC from 2000 to 2019. Test results were linked to medical visits, hospitalizations, cancers, prescription drugs, and mortality data. Six HCV care cascade stages were identified: (1) antibody diagnosed; (2) RNA tested; (3) RNA positive; (4) genotyped; (5) initiated treatment; and (6) achieved sustained virologic response (SVR). HCV care cascade results were assessed for women, and an 'inverse' cascade was created to assess gaps, including not being RNA tested, genotyped, or treatment initiated, stratified by sex.

Results: In 2019, 52,638 people with known sex were anti-HCV positive in BC; 37\% $(19,522)$ were women. Confirmatory RNA tests were received by $86 \%(16,797 / 19,522)$ of anti-HCV positive women and $83 \%(27,353 / 33,116)$ of men. Among people who had been genotyped, $68 \%(6756 / 10,008)$ of women and $67 \%(12,640 / 18,828)$ of men initiated treatment, with 94\% (5023/5364) of women and 92\% (9147/9897) of men achieving SVR. Among the 3252 women and 6188 men not yet treated, higher proportions of women compared to men were born after 1975 (30\% vs. 21\%), had a mental health diagnosis (42\% vs. 34\%) and had used injection drugs (50\% vs. 45\%). Among 1619 women and 2780 men who had used injection drugs and were not yet treated, higher proportions of women than men used stimulants (64\% vs. $57 \%)$, and opiates (67\% vs. $60 \%)$.
\end{abstract}

Conclusions: Women and men appear to be equally engaged into the HCV care cascade; however, women with concurrent social and health conditions are being left behind. Treatment access may be improved with approaches that meet the needs of younger women, those with mental health diagnoses, and women who use drugs.

*Correspondence: margo.pearce@bccdc.ca

${ }^{2}$ School of Population and Public Health, University of British Columbia (UBC), Vancouver, BC, Canada

Full list of author information is available at the end of the article

\section{Introduction}

The treatment experiences and needs of women living with hepatitis $\mathrm{C}$ virus $(\mathrm{HCV})$ are frequently overlooked in research, yet there are relevant clinical differences between men and women related to $\mathrm{HCV}$ infection and 
disease progression. Female sex is a significant predictor for spontaneous clearance among people with acute $\mathrm{HCV}$ infection and a factor in liver disease progression among those living with chronic HCV [1]. Rates of liver fibrosis and cirrhosis progression appear to be slower in younger women ( $<50$ years) compared to men; however, this difference disappears in older women ( $>50$ years), possibly due to hormonal changes in menopause $[1,2]$. For women who have reproductive potential, HCV in pregnancy is a concern. Though pregnancy does not exacerbate $\mathrm{HCV}$ disease progression, $\mathrm{HCV}$ infection can contribute to adverse perinatal outcomes [3-6]. In addition, vertical HCV transmission affects $4-7 \%$ of infants born to women living with chronic HCV and up to $11 \%$ of infants born to women with HIV-HCV co-infection [7]. There are also gendered differences that underscore intersectional barriers faced by some women living with HCV. A cohort study in Ontario, Canada, highlighted that compared to men, women's immigration status and lower socioeconomic status were more likely to negatively affect $\mathrm{HCV}$ treatment uptake [2]. Other studies have reported barriers to women's access to HCV treatment, including advanced age, rurality, injection drug use, and involvement in sex work [8-12].

The introduction of novel direct-acting antiviral (DAA) therapies for chronic $\mathrm{HCV}$ infection has dramatically changed the $\mathrm{HCV}$ treatment landscape. In contrast with the arduous and moderately effective interferonbased treatment, DAA therapies are a well-tolerated and highly effective cure, with $>95 \%$ of patients achieving sustained virological response (SVR) in just 8-12 weeks [13]. Reduced clinical barriers to HCV cure have inspired the possibility of eliminating HCV globally by 2030 , which will require diagnosing $>80 \%$ of those living with $\mathrm{HCV}$ and treating $85 \%$ of those diagnosed with chronic infection [14]. To achieve this, new HCV infections and deaths related to $\mathrm{HCV}$ need to be addressed alongside DAA treatments through scaled-up harm reduction and linkage to liver care.

A powerful way to monitor progress toward $\mathrm{HCV}$ elimination goals is to evaluate the $\mathrm{HCV}$ care cascade at the population level by assessing progress through RNA testing, genotype testing, treatment initiation, and SVR stages. In British Columbia (BC), Canada, integrated population-level laboratory testing and health administration data has made this possible. A 2018 analysis demonstrated that women comprised $37 \%$ of the approximately 53,000 people living with $\mathrm{HCV}$ in $\mathrm{BC}$ and that similar proportions of men and women progressed through the stages of care [15]. However, little is understood regarding factors that influence women's access to HCV care at the population level. These factors may compound existing barriers and create, or negatively contribute to, risk environments where women are "hardly reached" by health/social services and at greater risk for adverse health outcomes [16]. Monitoring of $\mathrm{HCV}$ diagnosis and care among women is thus critical both to achieve $\mathrm{HCV}$ elimination goals in $\mathrm{BC}$ and ensure that women receive timely and equitable access.

The objectives of this study were to: (a) construct the population-level $\mathrm{HCV}$ care cascade in $\mathrm{BC}$ stratified by sex from 2000 to 2019; (b) evaluate progress through the stages of the $2019 \mathrm{HCV}$ care cascade for women and men living with $\mathrm{HCV}$ in $\mathrm{BC}$; (c) characterize progress and highlight gaps in the $\mathrm{HCV}$ care cascade experienced by women living with $\mathrm{HCV}$ in $\mathrm{BC}$.

\section{Methods}

This study represents population data from BC, Canada, where all residents are registered for publicly funded health insurance via the Medical Services Plan (MSP). MSP is a single-payer system covering healthcare provided by fee-for-service practitioners including general practices, private laboratories, and other providers. Laboratory $\mathrm{HCV}$ testing for the entire province is centralized at the $\mathrm{BC}$ Centre for Disease Control Public Health Laboratory (BCCDC PHL) except for $5 \%$ of tests that are performed at regional labs, which send specimens that test positive to BCCDC PHL for confirmation. All prescriptions dispensed in $\mathrm{BC}$ are recorded within a central payer-agnostic system called PharmaNet.

HCV therapies are publicly funded in BC through the PharmaCare Limited Coverage Drug Program. Interferon-based combination therapies (Interferon/Ribavirin) for HCV treatment became available in 2000, and the more efficacious Pegylated interferon/Ribavirin therapy became available in May 2003 [17]. DAA treatments were available in BC in 2014 and became publicly funded in early 2015, though eligibility for public coverage was restricted to priority patients with fibrosis stage 2 (F2) or above (Metavir or equivalent) or extrahepatic manifestations. In March 2017, eligibility for public coverage expanded to people with comorbidities including HIV or hepatitis B (HBV) co-infection, diabetes, chronic kidney disease, co-existent liver disease, and women who were planning to become pregnant in the next 12 months [18]. Remaining restrictions for publicly funded DAA treatment were removed in BC in April 2018. HCV testing and treatment in $\mathrm{BC}$ is provided in various healthcare settings including primary, community, and specialized clinics. It is important to note that prior to January 2020, new HCV antibody positive tests required a follow-up EDTA blood sample for HCV RNA nucleic acid testing (NAT). As of January 2020, persons who are positive for anti-HCV antibodies will automatically be tested for HCV RNA by NAT if: (1) they are first-time antibody positive or (2) if 
they have not been tested by NAT before. HCV genotype testing is required to prescribe $\mathrm{HCV}$ treatment in $\mathrm{BC}$.

This analysis uses data from the British Columbia Hepatitis Testers Cohort (BC-HTC) study. We have previously published on the $\mathrm{BC}$-HTC construction and data linkage [19]. Briefly, BC-HTC includes all BC residents who ever tested for HCV or HIV, or were diagnosed with HBV, HCV, HIV, or active tuberculosis (TB) in BC between 1990 and 2015, linked with data on medical visits, hospitalizations, cancers, prescription drugs, and deaths. The laboratory, prescription, and mortality data were updated to 31 December 2019 to facilitate creation and assessment of the $2019 \mathrm{HCV}$ care cascade (Additional file 1: Table S1). In this study, we refer to 'women' as people who were assigned female sex at birth. Although 'woman' also implies gender identity, this was not determinable in this study.

BC-HTC data are de-identified and analyzed anonymously; thus, informed consent was not required. Institutional ethics approval was provided by the University of British Columbia Research Ethics Board (H14-01649) and all research was carried out in accordance with relevant guidelines and regulations.

\section{Cascade of HCV care}

Operational definitions for six stages of the $\mathrm{HCV}$ cascade of care are described in Additional file 1: Table S2. The stages were defined as: a) HCV diagnosed; (b) HCV RNA tested; (c) HCV RNA positive; (d) genotyped; (e) initiated antiviral treatment; and (f) sustained viral response (SVR). We applied these definitions to the data to estimate the number and proportion of women in each stage by the end of the year from 2000 to 2019. Focusing on the year 2019, we also applied these definitions to compare the number and proportion of both men and women at each stage. Next, we evaluated the 2019 cascade stages by demographic characteristics and comorbidity profiles of women who were diagnosed with HCV. Finally, to get a clearer understanding of gaps and leakage in the $\mathrm{HCV}$ care cascade among women compared to men, we report on the inverse 2019 HCV care cascade: the number and proportions of women and men who were diagnosed anti-HCV positive but did not advance to HCV RNA testing, genotype testing, or treatment initiation stages.

\section{Estimate of viraemia}

The estimate of HCV RNA positive women in BC in 2019 was based on: (1) the number of untreated women whose last HCV RNA test on record is positive; (2) 75\% [20] of those who were positive by antibody testing and had no HCV RNA or genotype testing done, as about $25 \%$ of antibody-positive people clear infection spontaneously; (3) $75 \%$ [21] of the untested and undiagnosed estimate; (4) those treated women determined not to have achieved SVR (the SVR rate calculated for treated women with available RNA test after treatment was used to estimate how many treated women with no available RNA test after treatment would fail to achieve SVR) [15].

\section{Demographic characteristics and comorbidity profiles}

Demographic characteristics included birth cohort, ethnicity, social and material deprivation [22], and urbanicity. Ethnicity was derived using Onomap software, which identifies ethnicity using name network cultural/linguistic clustering techniques [23-25]. Onomap has been previously validated and used in demographic and health research $[23,24]$. Onomap is prone to misclassifying people with anglicized names and those with mixed ethnicities [26]; however, our internal validation demonstrated that Onomap's sensitivity and specificity relative to self-identified ethnicity was $93 \%$ and $98.6 \%$ for South Asian people, respectively, and $66.7 \%$ and $99.5 \%$ for East Asian people, respectively. Ethnic groups were therefore classified as South Asian, East Asian, and Other BC Residents. Comorbidity indicators were derived from MSP data containing physician fee-for-service billing and diagnostic codes, and hospitalization data for mental health diagnoses, problematic alcohol and drug use, cirrhosis, and decompensated cirrhosis (Additional file 1: Table S3).

Characteristics and comorbidities of people diagnosed $\mathrm{HCV}$ antibody-positive were stratified by sex as well as proportions of women and men at each stage of the HCV care cascade. Chi-squared tests were carried out to compare categorical variables between women and men. All analyses were conducted using SAS/STAT software version 9.4 and $\mathrm{R}$ version 3.4.3.

\section{Role of the funding source}

The BC Centre for Disease Control supported construction of the BC-HTC to inform policy and program related to $\mathrm{HCV}$ in $\mathrm{BC}$. The study's funders had no role in study design, data analysis, data interpretation, or writing of the article.

\section{Patient and public involvement}

In Spring 2020, study investigators engaged with a community-based $\mathrm{HIV} / \mathrm{HCV}$ organization in $\mathrm{BC}$ and a group of women with lived experience (WWLE) of the HCV care cascade to prioritize lines of inquiry. Over the next eight weeks, through a collaborative, consensus-based process, we reviewed results with the group of WWLE to take into account their perspectives and feedback and ensure findings were interpreted in ways that were destigmatizing and relevant to communities. This work culminated in two open-access 90-minute webinars in Summer 
2020 that focused on the WWLE's reflections and policy recommendations in response to the study results [27].

\section{Results}

Women in the HCV care cascade 2000-2019

Figure 1 displays women in the $\mathrm{HCV}$ cascade of care in BC from 2000 to 2019. The numbers of estimated and diagnosed anti-HCV positive women increased substantially from 2000 (15,305 and 11,479, respectively), reaching peaks in 2015 (24,710 and 20,062, respectively) followed by slight declines towards 2019 (22,056 and 19,522, respectively), which may be an artifact of the 2015 cohort data rather than fewer diagnoses. The number of antibody-positive women who underwent confirmatory HCV RNA testing also increased over time from $1987(17.3 \%)$ in 2000 to 16,874 (84.9\%) in 2017, followed by a slight decline to $16,797(86 \%)$ in 2019 . Coinciding with more women being HCV RNA tested, there was an increasing number of anti-HCV positive women with confirmed HCV RNA positive test results (i.e. chronic HCV infection) over the study period, from 1552 (78.1\%) in 2000 to 11,075 (65.9\%) in 2019. The number of women with chronic $\mathrm{HCV}$ infection who received a genotype test also increased substantially over the study period from $673(43.4 \%)$ in 2000 to 10,007 (90.9\%) in 2019. In 2000, just 572 of genotyped women initiated HCV treatment, and this number increased steadily over time, reaching $6755(67.5 \%)$ in 2019. There was a corresponding increase in the number of women with known PCR information who achieved SVR after treatment, from 53\% (279/531) in 2012 after Pegylated interferon/Ribavirin became available, to $79 \%(1,893 / 2,393)$ in 2015 when DAAs were offered to priority patients, to $94 \%(4,932 / 5,465)$ in 2019 when DAAs were available to all people in $\mathrm{BC}$ living with $\mathrm{HCV}$.

\section{Estimated prevalence of anti-HCV positive women and viraemic proportion}

The estimated prevalence of anti-HCV positive women in BC (including untested and undiagnosed women) declined from $25,251(0.6 \%)$ in 2012 to $22,056(0.4 \%)$ in 2019 (Additional file 1: Table S4). The estimated number of women who were viraemic also declined from 16,250 to $2012(0.4 \%)$ to $8677(0.2 \%)$ in 2019 . The corresponding rate of viraemia declined from $64.4 \%$ of anti-HCV positive women in 2012 to $39.3 \%$ in 2019.

\section{The $2019 \mathrm{HCV}$ care cascade for women and men in BC}

Figure 2 displays the $2019 \mathrm{HCV}$ care cascade for women (light blue) and men (dark blue) in BC. In 2019, 52,638 people with known sex, including 19,522 women and 33,116 men were anti-HCV positive in $2019(n=3$ people had unknown sex). The proportion of anti-HCV positive women receiving a confirmatory RNA test was $86 \%$ $(16,797 / 19,522)$ compared to $82.6 \%(27,353 / 33,116)$ of men. $34 \%(5721 / 16,797)$ of women RNA tested had negative results (spontaneously cleared) compared to $24.1 \%$ $(6602 / 27,353)$ of men. Among people who had the virus they acquired genotyped, $68 \%(6756 / 10,008)$ of women and $67 \%(12,640 / 18,828)$ of men initiated treatment, with

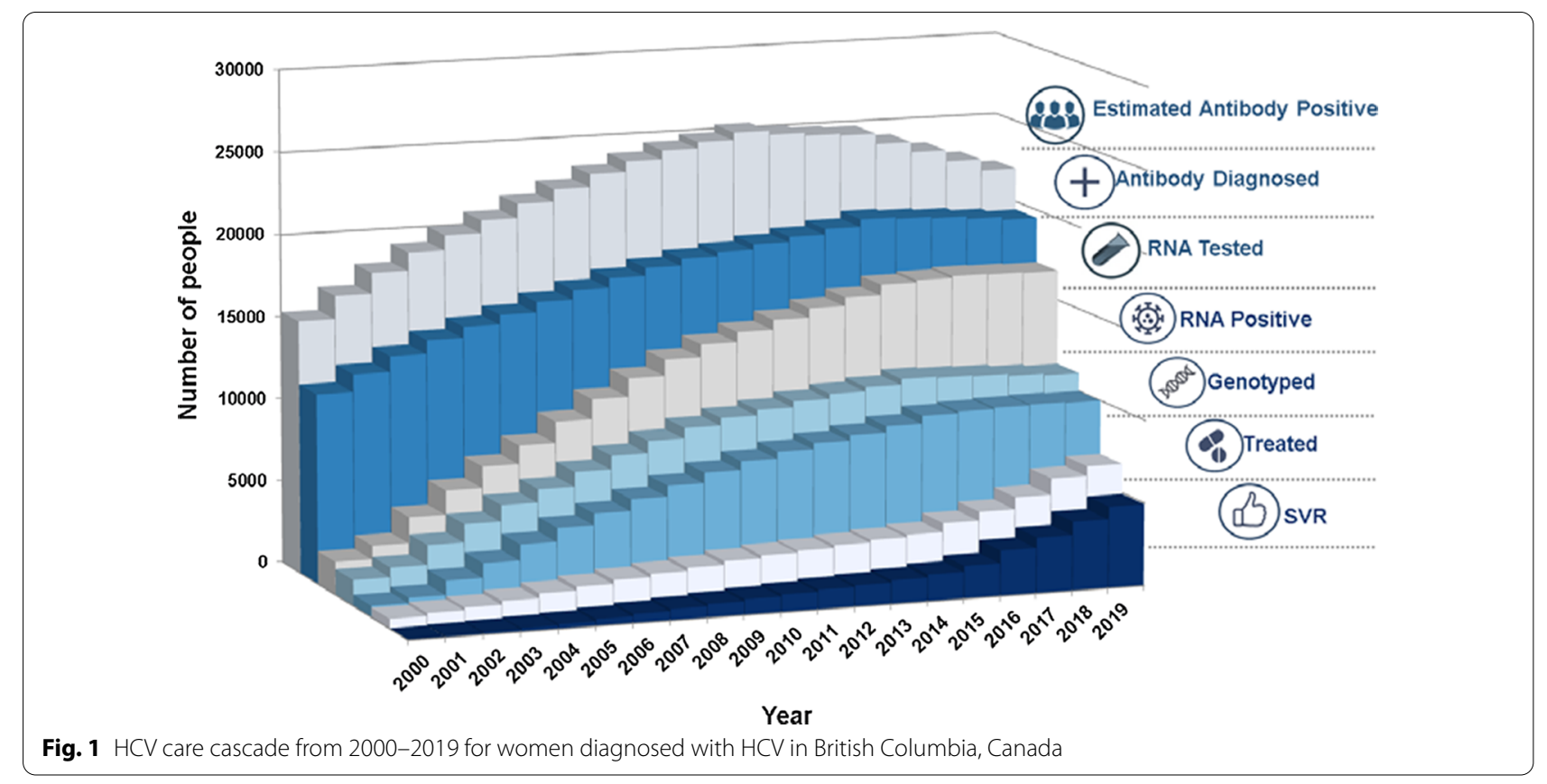




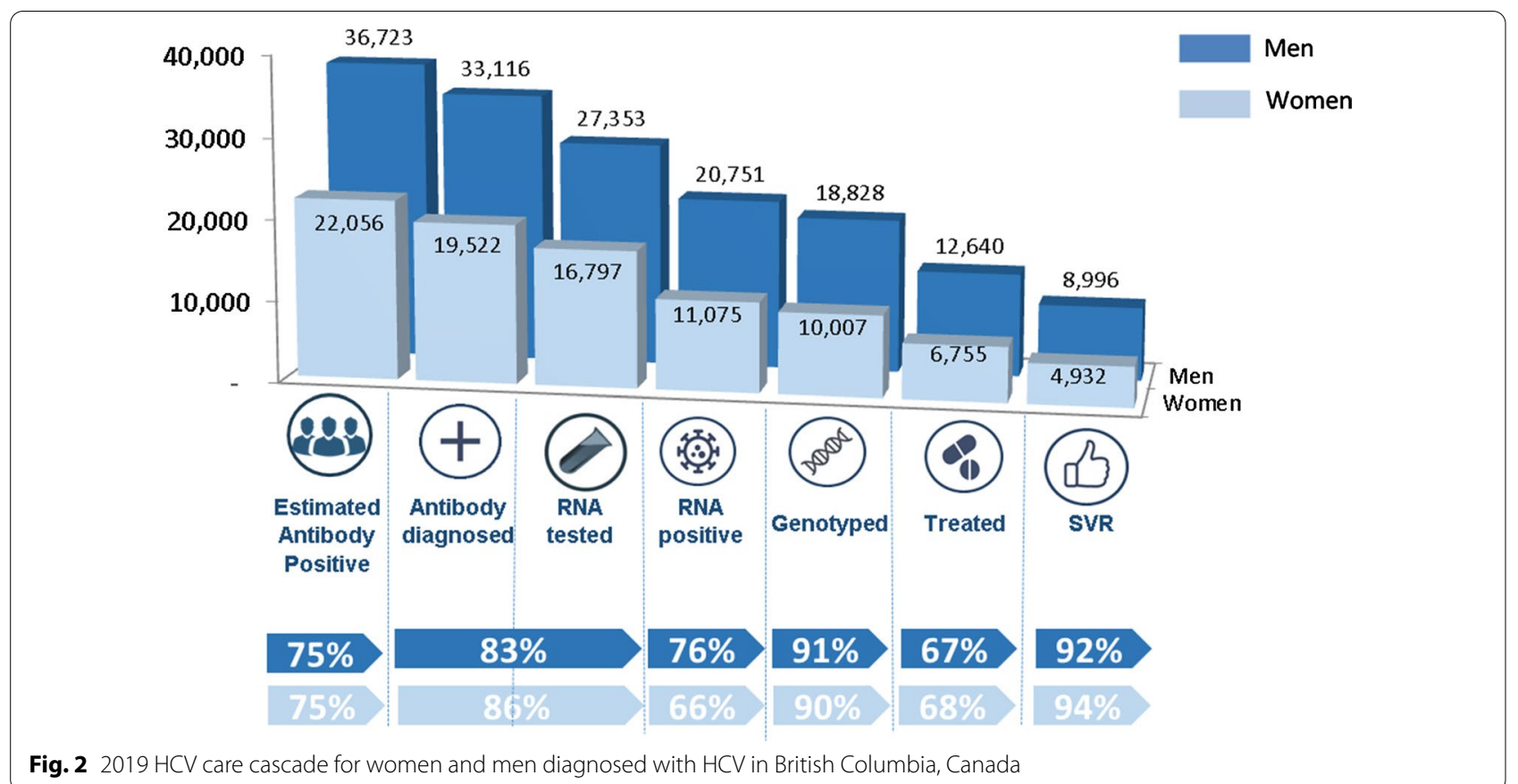

94\% (5023/5364) of women and 92\% (9147/9897) of men achieving SVR.

\section{Characteristics and comorbidity profiles of HCV antibody-positive women in 2019 , stratified by cascade of care stages}

Characteristics and comorbidity profiles of women at each stage of the $2019 \mathrm{HCV}$ care cascade are displayed in Table 1. Women born within the 1945-1964 birth cohort represented the highest proportion of all anti$\mathrm{HCV}$ positive women (51.5\%) and in each stage of the care cascade thereafter, including $56.2 \%$ of those genotyped, $63.5 \%$ of those who initiated HCV treatment, and $66.5 \%$ of those who achieved SVR. Younger women born between 1965 and 1974 and $\geq 1975$ were 21.3 and $22.2 \%$ of all anti-HCV positive women, respectively, yet smaller proportions of those women progressed through the care cascade, making up just 17.7 and $14 \%$ of women who initiated HCV treatment, and 16.5 and $12.4 \%$ of women who achieved SVR, respectively. Women with East Asian or South Asian ethnicity comprised 5.4 and $4.5 \%$ of anti$\mathrm{HCV}$ positive women in $\mathrm{BC}$, with proportions increasing slightly as the women progressed through the care cascade stages, reaching 5.6 and $5.9 \%$ of women who initiated HCV treatment and 6.1 and $5.3 \%$ of women who achieved SVR, respectively. Women within the most materially deprived quintile (Q5) made up a consistent proportion in each cascade stage, including $27.6 \%$ of those anti-HCV diagnosed, $27.8 \%$ of those RNA tested, $26.9 \%$ of those genotyped, $24.6 \%$ of those who initiated treatment, and $23.7 \%$ of those who achieved SVR. The proportions of women in the cascade stages who were in the most socially deprived quintile (Q5) increased from $22 \%$ of those anti-HCV diagnosed and RNA tested to $35.9 \%$ of those genotyped, $32.9 \%$ of those who initiated treatment, and $32.3 \%$ of those who achieved SVR.

Women with a history of injection drug use made up $37.2 \%$ of women who were anti-HCV positive, $38.7 \%$ of women who were RNA tested, and $37.4 \%$ of women who were genotype tested, respectively; yet, they made up just $31.4 \%$ of women who initiated treatment and $28.6 \%$ of women who achieved SVR. Women with a history of injection drug use and who had used opioids made up about two-thirds of women in each stage of the $\mathrm{HCV}$ care cascade. Women with a history of injection drug use and who had used stimulants made up 59.5\% of antiHCV positive women, $60.6 \%$ of those RNA tested, $59.8 \%$ of those genotyped, and $56.6 \%$ of those initiated on treatment. The proportions of women in each stage of the cascade who had been diagnosed with a major mental health disorder were fairly consistent, including $37.8 \%$ of those anti-HCV positive, $39.6 \%$ of those RNA tested, $39.4 \%$ of those genotyped, $38.1 \%$ of those who started treatment, and $36.6 \%$ of those who achieved SVR. Women with $\mathrm{HCV} / \mathrm{HBV}$ co-infection made up $4.6 \%$ of women who were anti-HCV positive, $4.3 \%$ of those RNA tested and genotyped, $4.4 \%$ of those treatment initiated, and $4.6 \%$ of those who achieved SVR. Women with HCV/HIV coinfection made up $2.9 \%$ of anti-HCV positive women, were $3.1 \%$ of those who were RNA tested, and $3.3 \%$ of 
Table 1 Demographic characteristics and comorbidity profile for women diagnosed with HCV in BC in 2019, stratified by care cascade stages

\begin{tabular}{|c|c|c|c|c|c|c|c|c|c|c|c|c|c|c|}
\hline \multirow[t]{2}{*}{ Variable } & \multicolumn{2}{|c|}{$\begin{array}{l}\text { Antibody } \\
\text { Diagnosed }\end{array}$} & \multicolumn{2}{|c|}{ HCV RNA Tested } & \multicolumn{2}{|c|}{$\begin{array}{l}\text { HCV RNA } \\
\text { Positive }\end{array}$} & \multicolumn{2}{|c|}{ HCV Genotyped } & \multicolumn{2}{|c|}{$\begin{array}{l}\text { HCV } \\
\text { Treatment } \\
\text { Initiated } \\
\end{array}$} & \multicolumn{2}{|c|}{$\begin{array}{l}\text { SVR Achieved/ } \\
\text { Unknown }\end{array}$} & \multicolumn{2}{|c|}{$\begin{array}{l}\text { SVR } \\
\text { Achieved }\end{array}$} \\
\hline & $\mathrm{n}$ & $\%$ & $n$ & $\%$ & $n$ & $\%$ & $n$ & $\%$ & $\mathrm{n}$ & $\%$ & $\mathrm{n}$ & $\%$ & $n$ & $\%$ \\
\hline Total & 19,522 & 100 & 16,797 & 100 & 11,075 & 100 & 10,007 & 100 & 6755 & 100 & 5683 & 100 & 4932 & 100 \\
\hline \multicolumn{15}{|l|}{ Birth Cohort } \\
\hline$<1945$ & 974 & 4.99 & 786 & 4.68 & 570 & 5.15 & 505 & 5.05 & 322 & 4.77 & 270 & 4.75 & 228 & 4.62 \\
\hline 1945-1964 & 10,046 & 51.46 & 8701 & 51.8 & 6028 & 54.43 & 5620 & 56.16 & 4288 & 63.48 & 3680 & 64.75 & 3279 & 66.48 \\
\hline 1965-1974 & 4162 & 21.32 & 3492 & 20.79 & 2210 & 19.95 & 1975 & 19.74 & 1197 & 17.72 & 986 & 17.35 & 814 & 16.5 \\
\hline$>1974$ & 4340 & 22.23 & 3818 & 22.73 & 2267 & 20.47 & 1907 & 19.06 & 948 & 14.03 & 747 & 13.14 & 611 & 12.39 \\
\hline \multicolumn{15}{|l|}{ Ethnicity } \\
\hline East Asian & 1045 & 5.35 & 864 & 5.14 & 542 & 4.89 & 496 & 4.96 & 379 & 5.61 & 331 & 5.82 & 302 & 6.12 \\
\hline South Asian & 872 & 4.47 & 803 & 4.78 & 549 & 4.96 & 521 & 5.21 & 395 & 5.85 & 338 & 5.95 & 263 & 5.33 \\
\hline Other BC Residents & 17,605 & 90.2 & 15,130 & 90.1 & 9984 & 90.15 & 8990 & 89.84 & 5981 & 88.54 & 5014 & 88.22 & 4367 & 88.55 \\
\hline \multicolumn{15}{|c|}{ Material Deprivation Quintile } \\
\hline Q1 (most privileged) & 2622 & 13.43 & 2294 & 13.66 & 1514 & 13.67 & 1390 & 13.89 & 953 & 14.11 & 797 & 14.02 & 698 & 14.15 \\
\hline Q2 & 2985 & 15.29 & 2602 & 15.49 & 1731 & 15.63 & 1597 & 15.96 & 1140 & 16.88 & 958 & 16.86 & 844 & 17.11 \\
\hline Q3 & 3557 & 18.22 & 3085 & 18.37 & 2058 & 18.58 & 1859 & 18.58 & 1337 & 19.79 & 1135 & 19.97 & 995 & 20.17 \\
\hline Q4 & 4407 & 22.57 & 3778 & 22.49 & 2511 & 22.67 & 2289 & 22.87 & 1569 & 23.23 & 1351 & 23.77 & 1155 & 23.42 \\
\hline Q5 (most deprived) & 5391 & 27.61 & 4669 & 27.8 & 3033 & 27.39 & 2689 & 26.87 & 1662 & 24.6 & 1361 & 23.95 & 1168 & 23.68 \\
\hline Unknown & 560 & 2.87 & 369 & 2.2 & 228 & 2.06 & 183 & 1.83 & 94 & 1.39 & 81 & 1.43 & 72 & 1.46 \\
\hline \multicolumn{15}{|c|}{ Social Deprivation Quintile } \\
\hline Q1 (most privileged) & 2351 & 12.04 & 369 & 2.2 & 1369 & 12.36 & 1258 & 12.57 & 923 & 13.66 & 766 & 13.48 & 642 & 13.02 \\
\hline Q2 & 2337 & 11.97 & 2053 & 12.22 & 1320 & 11.92 & 1209 & 12.08 & 878 & 13 & 768 & 13.51 & 648 & 13.14 \\
\hline Q3 & 2992 & 15.33 & 2018 & 12.01 & 1749 & 15.79 & 1591 & 15.9 & 1113 & 16.48 & 943 & 16.59 & 838 & 16.99 \\
\hline Q4 & 4332 & 22.19 & 2599 & 15.47 & 2392 & 21.6 & 2175 & 21.73 & 1522 & 22.53 & 1269 & 22.33 & 1137 & 23.05 \\
\hline Q5 (most deprived) & 6950 & 35.60 & 3717 & 22.13 & 4017 & 36.27 & 3591 & 35.88 & 2225 & 32.94 & 1856 & 32.66 & 1595 & 32.34 \\
\hline Unknown & 560 & 2.87 & 6041 & 35.96 & 228 & 2.06 & 183 & 1.83 & 94 & 1.39 & 81 & 1.43 & 72 & 1.46 \\
\hline \multicolumn{15}{|l|}{ Urbanicity } \\
\hline Rural & 2525 & 12.93 & 2222 & 13.23 & 1484 & 13.4 & 47 & 0.47 & 983 & 14.55 & 845 & 14.87 & 747 & 15.15 \\
\hline Urban & 16683 & 85.46 & 14436 & 85.94 & 9511 & 85.88 & 1379 & 13.78 & 5766 & 85.36 & 4835 & 85.08 & 4182 & 84.79 \\
\hline Unknown & 314 & 1.61 & 139 & 0.83 & 80 & 0.72 & 8581 & 85.75 & 6 & 0.09 & 3 & 0.05 & 3 & 0.06 \\
\hline \multicolumn{15}{|c|}{ History of Injection Drug Use } \\
\hline Yes & 7265 & 37.21 & 6497 & 38.68 & 4210 & 38.01 & 3741 & 37.38 & 2122 & 31.41 & 1698 & 29.88 & 1412 & 28.63 \\
\hline No & 12257 & 62.79 & 10300 & 61.32 & 6865 & 61.99 & 6266 & 62.62 & 4633 & 68.59 & 3985 & 70.12 & 3520 & 71.37 \\
\hline \multicolumn{15}{|l|}{ History of Opioid use } \\
\hline Yes & 4556 & 62.71 & 4154 & 63.94 & 2654 & 63.04 & 2375 & 63.49 & 1294 & 60.98 & 1021 & 60.13 & 843 & 59.7 \\
\hline No & 2709 & 37.29 & 2343 & 3606 & 1556 & 36.96 & 1366 & 36.51 & 828 & 39.02 & 677 & 39.87 & 569 & 40.3 \\
\hline \multicolumn{15}{|c|}{ History of stimulant use* } \\
\hline Yes & 4327 & 59.56 & 3938 & 60.61 & 2513 & 59.69 & 2238 & 59.82 & 1202 & 56.64 & 945 & 55.65 & 768 & 54.39 \\
\hline No & 2938 & 40.44 & 2559 & 39.39 & 1697 & 40.31 & 1503 & 40.18 & 920 & 43.36 & 753 & 44.35 & 644 & 45.61 \\
\hline \multicolumn{15}{|l|}{ Problematic Alcohol Use } \\
\hline Yes & 4952 & 25.37 & 4442 & 26.45 & 2797 & 25.26 & 2534 & 25.32 & 1549 & 22.93 & 1284 & 22.59 & 1084 & 21.98 \\
\hline No/Unknown & 14570 & 74.63 & 12355 & 73.55 & 8278 & 74.74 & 7473 & 74.68 & 5206 & 77.07 & 4399 & 77.41 & 3848 & 78.02 \\
\hline \multicolumn{15}{|c|}{ Major Mental Health Diagnosis } \\
\hline Yes & 7,379 & 37.8 & 6646 & 39.57 & 4331 & 39.11 & 3945 & 39.42 & 2572 & 38.08 & 2115 & 37.22 & 1804 & 36.58 \\
\hline No/Unknown & 12,143 & 62.2 & 10,151 & 60.43 & 6744 & 60.89 & 6062 & 60.58 & 4183 & 61.92 & 3568 & 62.78 & 3128 & 63.42 \\
\hline HBV Coinfection & & & & & & & & & & & & & & \\
\hline Yes & 905 & 4.64 & 904 & 5.38 & 579 & 5.23 & 538 & 5.38 & 366 & 5.42 & 575 & 5.42 & 487 & 9.87 \\
\hline No/Unknown & 18,617 & 95.36 & 15,893 & 94.62 & 10,496 & 94.77 & 9469 & 94.62 & 6389 & 94.58 & 5108 & 94.58 & 4445 & 90.13 \\
\hline
\end{tabular}


Table 1 (continued)

\begin{tabular}{|c|c|c|c|c|c|c|c|c|c|c|c|c|c|c|}
\hline \multirow[t]{2}{*}{ Variable } & \multicolumn{2}{|c|}{$\begin{array}{l}\text { Antibody } \\
\text { Diagnosed }\end{array}$} & \multicolumn{2}{|c|}{ HCV RNA Tested } & \multicolumn{2}{|c|}{$\begin{array}{l}\text { HCV RNA } \\
\text { Positive }\end{array}$} & \multicolumn{2}{|c|}{ HCV Genotyped } & \multicolumn{2}{|c|}{$\begin{array}{l}\text { HCV } \\
\text { Treatment } \\
\text { Initiated } \\
\end{array}$} & \multicolumn{2}{|c|}{$\begin{array}{l}\text { SVR Achieved/ } \\
\text { Unknown }\end{array}$} & \multicolumn{2}{|c|}{$\begin{array}{l}\text { SVR } \\
\text { Achieved }\end{array}$} \\
\hline & $\mathbf{n}$ & $\%$ & $\mathrm{n}$ & $\%$ & $\mathrm{n}$ & $\%$ & $\mathrm{n}$ & $\%$ & $\mathrm{n}$ & $\%$ & $\mathrm{n}$ & $\%$ & $\mathrm{n}$ & $\%$ \\
\hline \multicolumn{15}{|c|}{ HIV/AIDS Coinfection } \\
\hline Yes & 565 & 2.89 & 904 & 5.38 & 475 & 4.29 & 448 & 4.48 & 281 & 4.16 & 233 & 4.1 & 204 & 4.14 \\
\hline No/Unknown & 18,957 & 97.11 & 16,096 & 95.82 & 10,600 & 95.72 & 9559 & 95.53 & 6474 & 95.84 & 5450 & 95.9 & 4728 & 95.86 \\
\hline \multicolumn{15}{|l|}{ Cirrhosis } \\
\hline Yes & 807 & 4.13 & 752 & 4.48 & 608 & 5.49 & 581 & 5.81 & 493 & 7.3 & 418 & 7.36 & 379 & 7.68 \\
\hline No/Unknown & 18,715 & 95.87 & 16,045 & 95.52 & 10,467 & 94.51 & 9426 & 94.19 & 6262 & 92.7 & 5265 & 92.64 & 4553 & 92.32 \\
\hline \multicolumn{15}{|c|}{ Decompensated Cirrhosis } \\
\hline Yes & 468 & 2.4 & 423 & 2.52 & 331 & 2.99 & 308 & 3.08 & 245 & 3.63 & 205 & 3.61 & 187 & 3.79 \\
\hline No/Unknown & 19,054 & 97.6 & 16,374 & 97.48 & 10,744 & 97.01 & 9699 & 96.92 & 6510 & 96.37 & 5478 & 96.39 & 4745 & 96.21 \\
\hline
\end{tabular}

*Includes only women with a history of injection drug use

those genotyped, $3 \%$ of those treatment initiated, and 3\% of those who achieved SVR. Women with liver cirrhosis comprised $4.1 \%$ of anti-HCV positive females, and made up somewhat increasing proportions of those who were RNA tested (5.5\%), genotyped (5.8\%), initiated treatment $7.3 \%$, and achieved SVR (7.7\%).

\section{Characteristics and comorbidity profiles of antibody diagnosed men and women in 2019 in BC, stratified by inverse $\mathrm{HCV}$ care cascade stages (not RNA tested, not genotyped, not treated)}

Characteristics and comorbidity profiles of men and women within the inverse $2019 \mathrm{HCV}$ care cascade are presented in Table 2. Women born between 1945 and 1974 made up the majority of women who were not RNA tested (49.4\%), genotyped (38.2\%), or initiated on treatment (41\%); yet, men within the 1945-1974 birth cohort made up relatively greater proportions of men who were not RNA tested (58.7\%), genotyped (49.6\%), or initiated on treatment (49.1\%). Higher proportions of younger anti-HCV positive women born $\geq 1975$ than men were not RNA tested (19.2\% vs. $11.9 \%$, respectively), genotyped (33.7\% vs. $20.2 \%$, respectively), or initiated on treatment $(29.5 \%$ vs. $20.8 \%$, respectively). A higher proportion of anti-HCV positive East Asian women compared to East Asian men had not been RNA tested (6.6\% vs. $3.3 \%$, respectively) $(p<0.001)$, though similar proportions had not been genotyped ( $4.3 \%$ of women vs. $3.4 \%$ of men) or initiated on treatment (3.6\% of women vs. $2 \%$ of men). Higher proportions of anti-HCV positive women than men who had not been RNA tested were within the most deprived material quintiles $(p=0.034)$ whereas lower proportions of women than men who had not been RNA tested were within the most deprived social quintiles $(p=0.013)$.
Increasing proportions of women and men who used injection drugs were left behind in the $\mathrm{HCV}$ cascade of care stages. Higher proportions of women than men who had injected drugs had not been RNA tested $(28.2 \%$ vs. $24.1 \%$, respectively) $(p<0.001)$, genotyped $(43.9 \%$ vs. $37.1 \%$, respectively) $(p<0.001)$, or treated $(49.8 \%$ vs. $44.9 \%$, respectively). $(p<0.001)$ Corresponding disparities were observed among people with a history of injection drug use who had used opioids, with $59.5 \%$ of women compared to $53.2 \%$ of men not being genotyped $(p<0.034)$, and $66.8 \%$ of women compared to $59.6 \%$ of men not initiating treatment $(p<0.001)$. Among people with a history of injection drug use who had used stimulants, $58.6 \%$ of women compared to $54.3 \%$ of men had not been genotyped and $64 \%$ of women compared to $56.8 \%$ of men had not initiated treatment $(p<0.001)$. Higher proportions of anti-HCV diagnosed women with a mental health diagnosis compared to men with a mental health diagnosis had not been RNA tested (27\% vs. $19.1 \%$, respectively) $(p<0.001)$, genotyped $(36.1 \%$ vs. $29.5 \%$, respectively) $(p<0.001)$, or initiated on treatment ( $42.2 \%$ vs. $34 \%$, respectively) $(p<0.001)$.

\section{Discussion}

Using population-based $\mathrm{HCV}$ care cascade monitoring data, this study has described women within and outside the $\mathrm{HCV}$ care cascade in $\mathrm{BC}$, Canada. We observed steady progress across the care cascade among women living with $\mathrm{HCV}$, with a substantial increase in treatment uptake after the introduction of DAAs in 2015 and expanded coverage starting in 2017. This increase also led to a reduction in the estimated prevalence of viraemic women in the province, from $0.4 \%$ to 2000 to $0.2 \%$ in 2019 [15]. In 2019, nearly equal proportions of women and men progressed through the $\mathrm{HCV}$ care cascade. 
Table 2 Demographic characteristics and comorbidity profile for women diagnosed with HCV in BC in 2019, who did not progress to the next stage of HCV care

\begin{tabular}{|c|c|c|c|c|c|c|c|c|c|c|c|c|}
\hline & \multicolumn{4}{|c|}{ Anti-HCV positive } & \multirow[b]{3}{*}{$p$-value } & \multicolumn{4}{|c|}{ Not HCV RNA tested } & \multirow[b]{3}{*}{$p$-value } & \multirow{2}{*}{\multicolumn{2}{|c|}{$\begin{array}{l}\begin{array}{l}\text { Not } \\
\text { genotyped }\end{array} \\
\text { Women }\end{array}$}} \\
\hline & \multicolumn{2}{|c|}{ Women } & \multicolumn{2}{|l|}{ Men } & & \multicolumn{2}{|c|}{ Women } & \multicolumn{2}{|l|}{ Men } & & & \\
\hline & $n$ & $\%$ & $\mathrm{n}$ & $\%$ & & $\mathrm{n}$ & $\%$ & $\mathrm{n}$ & $\%$ & & $\mathrm{n}$ & $\%$ \\
\hline Total & 19,522 & 100 & 33,116 & 100 & & 2725 & 100 & 5763 & 100 & & 1068 & 100 \\
\hline \multicolumn{13}{|l|}{ Birth Cohort } \\
\hline$<1945$ & 974 & 5 & 1151 & 3.48 & $<0.001$ & 188 & 6.9 & 256 & 4.44 & $<0.001$ & 65 & 6.09 \\
\hline 1945-1964 & 10,046 & 51.46 & 19,797 & 59.78 & & 1345 & 49.36 & 3382 & 58.68 & & 408 & 38.2 \\
\hline 1965-1974 & 4162 & 21.32 & 7374 & 22.27 & & 670 & 24.59 & 1440 & 24.99 & & 235 & 22 \\
\hline$\geq 1975$ & 4340 & 22.23 & 4794 & 14.48 & & 522 & 19.16 & 685 & 11.89 & & 360 & 33.71 \\
\hline \multicolumn{13}{|l|}{ Ethnicity } \\
\hline East Asian & 1045 & 5.35 & 1128 & 3.41 & $<0.001$ & 181 & 6.64 & 190 & 3.3 & $<0.001$ & 46 & 4.31 \\
\hline South Asian & 872 & 4.47 & 1283 & 3.87 & & 69 & 2.53 & 128 & 2.22 & & 28 & 2.62 \\
\hline Other BC Residents & 17,605 & 90.2 & 30,705 & 92.72 & & 2475 & 90.82 & 5445 & 94.48 & & 994 & 93.07 \\
\hline \multicolumn{13}{|c|}{ Material deprivation quintile } \\
\hline Q1 (most privileged) & 2622 & 13.43 & 4912 & 14.83 & $<0.001$ & 328 & 12.04 & 777 & 13.48 & 0.034 & 124 & 11.61 \\
\hline Q2 & 2985 & 15.29 & 5018 & 15.15 & & 383 & 14.06 & 812 & 14.09 & & 134 & 12.55 \\
\hline Q3 & 3557 & 18.22 & 5851 & 17.67 & & 472 & 17.32 & 905 & 15.7 & & 199 & 18.63 \\
\hline Q4 & 4407 & 22.57 & 7025 & 21.21 & & 629 & 23.08 & 1165 & 20.22 & & 222 & 20.79 \\
\hline Q5 (most deprived) & 5391 & 27.61 & 8972 & 27.09 & & 722 & 26.5 & 1474 & 25.58 & & 344 & 32.21 \\
\hline Unknown & 560 & 2.87 & 1338 & 4.04 & & 191 & 7.01 & 630 & 10.93 & & 45 & 4.21 \\
\hline \multicolumn{13}{|c|}{ Social deprivation quintile } \\
\hline Q1 (most privileged) & 2351 & 12.04 & 3643 & 11 & $<0.001$ & 298 & 10.94 & 537 & 9.32 & 0.013 & 111 & 10.39 \\
\hline Q2 & 2337 & 11.97 & 3955 & 11.94 & & 319 & 11.71 & 612 & 10.62 & & 111 & 10.39 \\
\hline Q3 & 2992 & 15.33 & 4791 & 14.47 & & 393 & 14.42 & 751 & 13.03 & & 158 & 14.79 \\
\hline Q4 & 4332 & 22.19 & 6877 & 20.77 & & 615 & 22.57 & 1181 & 20.49 & & 217 & 20.32 \\
\hline Q5 (most deprived) & 6950 & 35.6 & 12,512 & 37.78 & & 909 & 33.36 & 2052 & 35.61 & & 426 & 39.89 \\
\hline Unknown & 560 & 2.87 & 1338 & 4.04 & & 191 & 7.01 & 630 & 10.93 & & 45 & 4.21 \\
\hline \multicolumn{13}{|l|}{ Urbanicity } \\
\hline Rural & 2525 & 12.93 & 4396 & 13.27 & 0.077 & 303 & 11.12 & 646 & 11.21 & 0.453 & 105 & 9.83 \\
\hline Urban & 16,683 & 85.46 & 27,699 & 83.64 & & 2247 & 82.46 & 4531 & 78.62 & & 930 & 87.08 \\
\hline Unknown & 314 & 1.61 & 1021 & 3.08 & & 175 & 6.42 & 586 & 10.17 & & 33 & 3.09 \\
\hline \multicolumn{13}{|c|}{ History of Injection Drug Use } \\
\hline Yes & 7265 & 37.21 & 11,005 & 33.23 & $<0.001$ & 768 & 28.18 & 1391 & 24.14 & $<0.001$ & 469 & 43.91 \\
\hline No & 12,257 & 62.79 & 22,111 & 66.77 & & 1957 & 71.82 & 4372 & 75.86 & & 599 & 56.09 \\
\hline \multicolumn{13}{|l|}{ History of Opioid use* } \\
\hline Yes & 4556 & 62.71 & 6370 & 57.88 & $<0.001$ & 402 & 47.66 & 688 & 49.46 & 0.200 & 279 & 59.49 \\
\hline No & 2709 & 37.29 & 4635 & 42.12 & & 366 & 52.34 & 703 & 50.54 & & 190 & 40.51 \\
\hline \multicolumn{13}{|c|}{ History of stimulant use ${ }^{*}$} \\
\hline Yes & 4327 & 59.56 & 6023 & 54.73 & $<0.001$ & 389 & 50.65 & 706 & 50.75 & 0.963 & 275 & 58.64 \\
\hline No & 2938 & 40.44 & 4982 & 45.27 & & 379 & 49.35 & 685 & 49.25 & & 194 & 41.36 \\
\hline \multicolumn{13}{|l|}{ Problematic Alcohol Use } \\
\hline Yes & 4952 & 25.37 & 8809 & 26.6 & 0.002 & 510 & 18.72 & 1172 & 20.34 & 0.080 & 263 & 24.63 \\
\hline No/Unknown & 14,570 & 74.63 & 24,307 & 73.4 & & 2215 & 81.28 & 4591 & 79.66 & & 805 & 75.37 \\
\hline \multicolumn{13}{|c|}{ Major Mental Health Diagnosis } \\
\hline Yes & 7379 & 37.8 & 9527 & 28.77 & $<0.001$ & 733 & 26.9 & 1101 & 19.1 & $<0.001$ & 386 & 36.14 \\
\hline No/Unknown & 12,143 & 62.2 & 23,589 & 71.23 & & 1992 & 73.1 & 4662 & 80.9 & & 682 & 63.86 \\
\hline HBV Coinfection & & & & & & & & & & & & \\
\hline Yes & 905 & 4.64 & 1968 & 5.94 & 0.288 & 183 & 6.72 & 395 & 6.85 & 0.933 & 41 & 3.84 \\
\hline
\end{tabular}


Table 2 (continued)

\begin{tabular}{|c|c|c|c|c|c|c|c|c|c|c|c|c|}
\hline & \multicolumn{4}{|c|}{ Anti-HCV positive } & \multirow[b]{3}{*}{$p$-value } & \multicolumn{4}{|c|}{ Not HCV RNA tested } & \multirow[b]{3}{*}{$p$-value } & \multirow{2}{*}{\multicolumn{2}{|c|}{$\begin{array}{l}\begin{array}{l}\text { Not } \\
\text { genotyped }\end{array} \\
\text { Women }\end{array}$}} \\
\hline & \multicolumn{2}{|c|}{ Women } & \multicolumn{2}{|l|}{ Men } & & \multicolumn{2}{|c|}{ Women } & \multicolumn{2}{|l|}{ Men } & & & \\
\hline & $\mathbf{n}$ & $\%$ & n & $\%$ & & $n$ & $\%$ & $\mathbf{n}$ & $\%$ & & $\mathrm{n}$ & $\%$ \\
\hline No/Unknown & 18,617 & 95.36 & 31,148 & 94.05 & & 2542 & 93.29 & 5368 & 93.14 & & 1027 & 96.16 \\
\hline \multicolumn{13}{|l|}{ HIV/AIDS Coinfection } \\
\hline Yes & 565 & 2.89 & 1826 & 5.51 & $<0.001$ & 61 & 2.24 & 200 & 3.47 & 0.014 & 27 & 2.53 \\
\hline No/Unknown & 18,957 & 97.11 & 31,290 & 94.48 & & 2664 & 97.76 & 5563 & 96.53 & & 1041 & 97.47 \\
\hline \multicolumn{13}{|l|}{ Cirrhosis } \\
\hline Yes & 807 & 4.13 & 1474 & 4.45 & 0.084 & 55 & 2.02 & 115 & 2 & 0.944 & 27 & 2.53 \\
\hline No/Unknown & 18,715 & 95.87 & 31,642 & 95.55 & & 2670 & 97.98 & 5648 & 98 & & 1041 & 97.47 \\
\hline \multicolumn{13}{|c|}{ Decompensated Cirrhosis } \\
\hline Yes & \multirow{2}{*}{$\begin{array}{l}468 \\
19,054 \\
\end{array}$} & 2.4 & 776 & 2.34 & 0.694 & 45 & 1.65 & 78 & 1.35 & 0.284 & 23 & 2.15 \\
\hline No/Unknown & & 97.6 & 32,340 & 97.66 & & 2680 & 98.35 & 5685 & 98.65 & & 1045 & 97.85 \\
\hline & \multicolumn{3}{|c|}{ Not Genotyped } & & & \multicolumn{5}{|c|}{ Not treatment initiated } & & \\
\hline & $\mathrm{Me}$ & & & & & Women & & & Men & & & \\
\hline & $\mathrm{n}$ & & $\%$ & $p$-value & & $\mathrm{n}$ & $\%$ & & $\mathbf{n}$ & $\%$ & & $p$-value \\
\hline Total & 19 & & 100 & & & 3252 & 100 & & 6188 & 100 & & \\
\hline Birth Cohort & & & & & & & & & & & & \\
\hline$<1945$ & 75 & & 3.9 & $<0.001$ & & 183 & 5.63 & & 195 & 3.15 & & $<0.001$ \\
\hline $1945-1964$ & 95 & & 49.56 & & & 1332 & 40.96 & & 3038 & 49.1 & & \\
\hline 1965-1974 & 50 & & 26.37 & & & 778 & 23.92 & & 1671 & 27 & & \\
\hline$\geq 1975$ & 38 & & 20.18 & & & 959 & 29.49 & & 1284 & 20.75 & & \\
\hline Ethnicity & & & & & & & & & & & & \\
\hline East Asian & 65 & & 3.38 & 0.621 & & 117 & 3.6 & & 124 & 2 & & $<0.001$ \\
\hline South Asian & 55 & & 2.86 & & & 126 & 3.87 & & 207 & 3.35 & & \\
\hline Other BC Residents & 18 & & 93.76 & & & 3009 & 92.53 & & 5857 & 94.65 & & \\
\hline Material Deprivation & tile & & & & & & & & & & & \\
\hline Q1 (most privileged) & 24 & & 12.69 & 0.825 & & 437 & 13.44 & & 891 & 14.4 & & 0.417 \\
\hline Q2 & 23 & & 12.27 & & & 457 & 14.05 & & 795 & 12.85 & & \\
\hline Q3 & 32 & & 17.11 & & & 522 & 16.05 & & 1009 & 16.31 & & \\
\hline Q4 & 39 & & 20.54 & & & 720 & 22.14 & & 1348 & 21.78 & & \\
\hline Q5 (most deprived) & 61 & & 31.88 & & & 1027 & 31.58 & & 1957 & 31.63 & & \\
\hline Unknown & 10 & & 5.51 & & & 89 & 2.74 & & 188 & 3.04 & & \\
\hline Social Deprivation Qui & & & & & & & & & & & & \\
\hline Q1 (most privileged) & 18 & & 9.83 & 0.946 & & 335 & 10.3 & & 587 & 9.49 & & 0.064 \\
\hline Q2 & 20 & & 10.56 & & & 331 & 10.18 & & 648 & 10.47 & & \\
\hline Q3 & 26 & & 13.88 & & & 478 & 14.7 & & 809 & 13.07 & & \\
\hline Q4 & 37 & & 19.6 & & & 653 & 20.08 & & 1208 & 19.52 & & \\
\hline Q5 (most deprived) & 78 & & 40.61 & & & 1366 & 42 & & 2748 & 44.41 & & \\
\hline Unknown & 106 & & 5.51 & & & 89 & 2.74 & & 188 & 3.04 & & \\
\hline Urbanicity & & & & & & & & & & & & \\
\hline Rural & 20 & & 10.87 & 0.305 & & 396 & 12.18 & & 702 & 11.34 & & 0.285 \\
\hline Urban & 16 & & 84.56 & & & 2815 & 86.56 & & 5361 & 86.64 & & \\
\hline Unknown & 88 & & 4.58 & & & 41 & 1.26 & & 125 & 2.02 & & \\
\hline History of Injection Dru & & & & & & & & & & & & \\
\hline Yes & 71 & & 37.13 & 0.003 & & 1619 & 49.78 & & 2780 & 44.93 & & $<0.001$ \\
\hline No & 12 & & 62.87 & & & 1633 & 50.22 & & 3408 & 55.07 & & \\
\hline
\end{tabular}


Table 2 (continued)

\begin{tabular}{|c|c|c|c|c|c|c|c|c|}
\hline & \multirow{2}{*}{\multicolumn{2}{|c|}{$\begin{array}{l}\text { Not Genotyped } \\
\text { Men }\end{array}$}} & \multirow[b]{3}{*}{$p$-value } & \multicolumn{4}{|c|}{ Not treatment initiated } & \multirow[b]{3}{*}{$p$-value } \\
\hline & & & & \multicolumn{2}{|c|}{ Women } & \multicolumn{2}{|l|}{ Men } & \\
\hline & $\mathbf{n}$ & $\%$ & & $\mathbf{n}$ & $\%$ & $\mathbf{n}$ & $\%$ & \\
\hline \multicolumn{9}{|c|}{ History of Opioid use* } \\
\hline Yes & 380 & 53.22 & 0.034 & 1081 & 66.77 & 1656 & 59.57 & $<0.001$ \\
\hline No & 334 & 46.78 & & 538 & 33.23 & 1124 & 40.43 & \\
\hline \multicolumn{9}{|c|}{ History of stimulant use $e^{*}$} \\
\hline Yes & 399 & 54.34 & 0.146 & 1036 & 63.99 & 1580 & 56.83 & $<0.001$ \\
\hline No & 326 & 45.66 & & 583 & 36.01 & 1200 & 43.17 & \\
\hline \multicolumn{9}{|c|}{ Problematic Alcohol Use } \\
\hline Yes & 540 & 28.08 & 0.041 & 985 & 30.29 & 2051 & 33.14 & 0.005 \\
\hline No/Unknown & 1383 & 71.92 & & 2267 & 69.71 & 4137 & 66.86 & \\
\hline \multicolumn{9}{|c|}{ Major Mental Health Diagnosis } \\
\hline Yes & 567 & 29.49 & 0.002 & 1373 & 42.22 & 2105 & 34.02 & $<0.001$ \\
\hline No/Unknown & 1356 & 70.51 & & 1879 & 57.78 & 4083 & 65.98 & \\
\hline \multicolumn{9}{|l|}{ HBV Coinfection } \\
\hline Yes & 82 & 4.26 & 0.729 & 172 & 5.29 & 266 & 4.3 & 0.084 \\
\hline No/Unknown & 1841 & 95.74 & & 3080 & 94.71 & 5922 & 95.7 & \\
\hline \multicolumn{9}{|c|}{ HIV/AIDS Coinfection } \\
\hline Yes & 43 & 2.24 & 0.793 & 167 & 5.14 & 313 & 5.06 & 0.971 \\
\hline No/Unknown & 1880 & 97.77 & & 3085 & 94.87 & 5875 & 94.94 & \\
\hline \multicolumn{9}{|l|}{ Cirrhosis } \\
\hline Yes & 46 & 2.39 & 0.817 & 88 & 2.71 & 185 & 2.99 & 0.435 \\
\hline No/Unknown & 1877 & 97.61 & & 3164 & 97.29 & 6003 & 97.01 & \\
\hline \multicolumn{9}{|c|}{ Decompensated Cirrhosis } \\
\hline Yes & 30 & 1.56 & 0.239 & 63 & 1.94 & 112 & 1.81 & 0.663 \\
\hline No/Unknown & 1893 & 98.44 & & 3189 & 98.06 & 6076 & 98.19 & \\
\hline
\end{tabular}

Cl confidence interval, HR Hazard ratio. Multivariable analysis included adjustment for sex, Dukes class for test series and TNM4-stage for validation series, differentiation grade (G1-2 vs. G3-4), and age (as continuous). Age and TNM-stage/Dukes-classification also remained as independent predictors of prognosis in the multivariable mode

These results should encourage public health programming and treatment providers that significant progress is being made to eliminate $\mathrm{HCV}$ infection in $\mathrm{BC}$. We also identified key groups of women being left behind in the care cascade: specifically, younger women were less likely to progress across the cascade stages compared to men of the same age, which may impact reproductive outcomes. Similarly, women with problematic substance use were less likely to receive treatment. These findings highlighted opportunities to adapt programming and clinical care plans to accommodate women's needs, as HCV risk environments and barriers to treatment frequently intersect with sex and gender-based realities.

This study demonstrated that in 2019, just over half of women diagnosed anti-HCV positive in $\mathrm{BC}$ were born between 1945 and 1964 (baby boomers) and that this birth cohort represented an increasing proportion of women in subsequent $\mathrm{HCV}$ care cascade stages. Women in the 1965-1974 birth cohort comprised a significant proportion of women who were RNA positive and of those treated for HCV. These findings support previous research demonstrating that though the overall rate of HCV infection in the 1945-1974 birth cohort is declining, this population still makes up the majority of prevalent $\mathrm{HCV}$ infections in $\mathrm{BC}$ and Canada and those in need of HCV treatment [14, 28, 29]. Baby boomers have thus been identified as a priority population in Canada's $\mathrm{HCV}$ elimination targets, and national testing guidelines are for one-time HCV screening of all Canadians born between 1945-1974 [14, 30]. Most HCV infections among baby boomers result from past exposure in medical settings or past injection drug use; however, they may be less likely to seek out testing and treatment due to a lack of HCV awareness, difficulty recalling past exposures, or stigma related to substance use [31]. In the inverse HCV care cascade, women and men born between 1945 and 1964 made up similarly higher proportions of those not RNA tested, genotyped, or treatment 
initiated. Women born between 1965 and 1974 made up about one quarter of women not RNA tested, genotyped, or treatment initiated. As previously discussed, for older women living with $\mathrm{HCV}$, the risk for accelerated liver fibrosis progression becomes a concern. Most younger women living with chronic $\mathrm{HCV}$ experience slower liver disease progression, including cirrhosis and hepatocellular carcinoma[32], but some biological studies suggest that post-menopausal women may lose the putative protective effect of estrogen on the liver due to a decline of estrogen levels in the post-menopausal period $[1,33]$. Older women who have unknowingly been living with HCV for decades and those who are aware of their $\mathrm{HCV}$ diagnosis but have not yet engaged in the HCV care cascade may be at risk for advanced liver disease. Promising interventions aimed at increasing HCV screening and linkage to $\mathrm{HCV}$ care among baby boomers have leveraged the utility of electronic health records by adding $\mathrm{HCV}$ status to routine patient maintenance reminders for healthcare providers, followed by coordinated linkage to HCV treatment [31]. Similar approaches that also work to reduce the stigma associated with $\mathrm{HCV}$ infection may serve to identify older women in $\mathrm{BC}$ who are unaware of their $\mathrm{HCV}$ status and encourage engagement in the care cascade [11].

Younger women born after 1975 comprised 22.2\% of anti-HCV positive women, yet made up successively lower proportions of women in each HCV care cascade stage in 2019. Conversely, in the inverse HCV care cascade, these women comprised higher proportions than men among those not RNA tested, genotyped, or initiated on treatment. This finding parallels a previous study using population laboratory surveillance data in BC that demonstrated a significant increase over time in the proportion of newly diagnosed $\mathrm{HCV}$ positive women within an age range of reproductive potential who were lost to follow-up for RNA and/or genotype testing - from $10.2 \%$ to 2008 to $24.3 \%$ in 2019 [34]. Similarly, a large cohort study involving Veterans Administration data in the United States that found younger women had significantly lower odds of receiving DAA treatment than younger men [35]. As mentioned above, there is risk for vertical transmission among younger women living with $\mathrm{HCV}$ who become pregnant. A number of population-based studies in the US have indicated that rising maternal and pediatric HCV prevalence is likely related to concomitant increasing opioid use among women of reproductive potential [3, 36-38]. In the BC Hepatitis Testers Cohort, $61 \%$ of women with chronic HCV infection who were born after 1975 had histories of injection drug use and opiate use, among whom $50 \%$ had not been treated for HCV as of 2019. Treating women before or between pregnancies is therefore essential, yet, considering gendered realities faced by women living with $\mathrm{HCV}$, they must be assured that they will receive individual and family support as part of their HCV care plan. Younger women with past or current substance use may avoid or delay both prenatal and HCV care because of potential stigma within healthcare towards people who use substances or the possibility of their children being apprehended due to child welfare concerns [39]. Younger women may also be managing competing health, social, and economic priorities and feel they must delay treatment [40]. Outside of pregnancy, HCV infection is a concern for women's health [41]. Because of younger women's typical slower progression of liver disease, healthcare providers may mistakenly not prioritize $\mathrm{HCV}$ treatment for younger women with chronic $\mathrm{HCV}$ infection. Awareness of the potential long and short-term extrahepatic manifestations of $\mathrm{HCV}$ infection and potential improvements in quality of life should be emphasized to both care providers and younger women living with $\mathrm{HCV}$.

The majority of anti-HCV positive women in each stage of the HCV care cascade were within the most severe quintiles for material and social deprivation. In the inverse $\mathrm{HCV}$ care cascade, similarly high proportions of anti-HCV positive women and men who were not RNA tested, genotyped, or initiated on treatment had severe material deprivation. Likewise, high and similar proportions of anti-HCV positive women and men who had not been RNA tested, genotyped, or initiated on treatment had severe social deprivation. Though HCV care and treatment in $\mathrm{BC}$ is publically available to all living with $\mathrm{HCV}$ through universal healthcare, poverty and social isolation intersect with multifaceted issues faced by women with HCV. Women living with HCV have reported navigating gender-based violence, racism in the healthcare system, and immigration processes while juggling work, childcare, and other competing priorities $[10,42,43]$. These situations can create complex obstacles to women's HCV care, wherein some groups of women become among those who are "hardly reached" by treatment providers $[15,16]$. It is important to note that although $\mathrm{HCV}$ positive women who have recently immigrated, who are Indigenous or Black, are involved in sex work, or unstably housed were not identifiable in our study, the experiences and healthcare needs of these key groups of women have been previously highlighted in research and must not be overlooked moving forward $[10,43,44]$. Awareness of barriers and expansion of specialized, women-centered approaches, such as culturallysafe HCV outreach and peer-support programming, are therefore essential [45].

Women in $\mathrm{BC}$ with a history of injection drug use made up $37.2 \%$ of women who were HCV antibody diagnosed, 
$38.7 \%$ who were RNA tested, and $37.4 \%$ who were genotype tested; yet, they made up just $31.4 \%$ of women who initiated treatment and $28.6 \%$ of women who achieved SVR. In the inverse HCV care cascade, somewhat higher proportions of women compared to men who not been RNA tested, genotyped, or initiated on treatment had injected drugs. The proportions of both women and men living with $\mathrm{HCV}$ and a history of injection drug use and opioid or stimulant use steadily increased across the inverse cascade stages. Nevertheless, disproportionately higher numbers of anti-HCV positive women who had used opioids or stimulants were left behind in the care cascade. These findings correspond to studies based in the United States that have reported a high frequency of opiate and stimulant use among women at risk of or living with $\mathrm{HCV}$ [46]. In other $\mathrm{BC}$ population-based analyses, uninterrupted opioid agonist therapy (OAT) was associated with higher likelihood HCV treatment uptake among people who inject drugs after adjusting for sex, yet stimulant use disorder was negatively associated with treatment uptake $[47,48]$. Research has also demonstrated that gendered power dynamics contribute higher $\mathrm{HCV}$ exposure risk for women, such as being second on the needle, requiring help to inject, and needing to negotiate harm reduction with risk for violence [49-51]. Women with lived experience of HCV have highlighted that intersecting experiences of sexism, racism, and discrimination toward women who use injection drugs create significant barriers to accessing healthcare, including addiction treatment [27, 43]. Involving $\mathrm{HCV}$-affected women who use drugs in the design and delivery of $\mathrm{HCV}$ screening, treatment, and harm reduction programming will result in innovative solutions that address these barriers and lead to more women engaging in the HCV care cascade and experiencing improved wellbeing beyond achieving SVR.

Overall, this study demonstrated that in 2019, 37.8\% of women and $28.8 \%$ of men who were diagnosed anti$\mathrm{HCV}$ positive in $\mathrm{BC}$ had had a mental health diagnosis. Anti-HCV positive women with a mental health disorder made up about $40 \%$ of women within each stage of the $\mathrm{HCV}$ care cascade and increasing proportions of women in each inverse HCV care cascade stage. Higher proportions of women compared to men who had not been RNA tested, genotyped, or initiated on treatment had received a mental health diagnosis. National self-reported data suggests that women in Canada are more likely than men to have had past and recent major depression and generalized anxiety [52] and more likely to perceive that their mental health care needs are not met [53]. Intervention research based in the United States and Australia has reported that patients with severe mental health diagnoses who received $\mathrm{HCV}$ care integrated with mental health care had a higher likelihood of achieving SVR [54]. Few of the study participants were women, however, and therefore the relevance and effectiveness of such interventions for women who have mental illness and are living with $\mathrm{HCV}$ is unclear. In addition, mental health disorders are frequently concurrent with problematic substance use, requiring specialized care and harm reduction. Womencentred HCV interventions that are trauma-informed, culturally safe, and work within peer-support frameworks may better meet the needs of women diagnosed with mental health disorders [55].

We found that the proportion of women in each stage of the $2019 \mathrm{HCV}$ care cascade living with HCV-HBV co-infection was relatively constant at about $4.5 \%$. In the inverse cascade, proportions of women and men with $\mathrm{HCV}-\mathrm{HBV}$ co-infection who were not RNA tested were somewhat higher than the proportions who were not genotyped or initiated on treatment, highlighting that those who received RNA testing are likely to progress through subsequent HCV care cascade stages. Somewhat higher proportions of men compared to women in the inverse cascade stages were living with HCV-HIV co-infection, likely reflecting the higher burden of HIV infection among men in BC. Proportions of women and men living with $\mathrm{HCV}$ who had cirrhosis and decompensated cirrhosis were similar. It is important to note that although more prevalent among men living with $\mathrm{HCV}$, over $25 \%$ of women not HCV RNA tested, genotyped, or treated had problematic alcohol use. Accelerated liver disease progression among these women is of grave concern, especially among those unaware of their HCV diagnosis or treatment options. Continued focus on providing $\mathrm{HCV}$ treatment to women living with significant comorbidities is needed, specifically with enhanced models that address relational and contextual barriers to engaging in healthcare among women with $\mathrm{HCV}$ and HBV or HIV co-infection.

\section{Limitations}

Although this study is based on comprehensive data to characterize the $\mathrm{HCV}$ cascade of care in $\mathrm{BC}$, there are limitations that impact the measurement of each stage. The model to estimate the number of people who were undiagnosed $\mathrm{HCV}$ antibody-positive was based on 2012 $\mathrm{BC}$ and Canadian data [21, 56]. BC residents have historically tested for $\mathrm{HCV}$ more than other provinces, with testing volumes increasing in recent years, especially after the STOP-HIV initiative began in BC, suggesting that our estimate of the proportion who are undiagnosed may be lower than the national average. Further, the national mandate to test all baby boomers for HCV has increased the number of people born between 1945 and 1965 living with $\mathrm{HCV}$ infection who have been diagnosed; 
subsequently, $\mathrm{HCV}$ positivity is declining in this age group. Simultaneously, the number of new/incident cases of $\mathrm{HCV}$ have fallen in $\mathrm{BC}$ over the past decade, mortality among people with chronic $\mathrm{HCV}$ is higher compared to people without $\mathrm{HCV}$, and uptake of curative DAA treatments is increasing [57]. This study may therefore overestimate the number of undiagnosed and prevalent cases of HCV in BC; however, the estimated fraction of undiagnosed people in our cohort was similar to what Hamadeh et al. (2020) reported in population model estimates of chronic HCV infection in the province (33.3\%) [58]. In addition, BC-HTC data does not contain information about gender identity, and therefore we cannot comment on the HCV care cascade experienced by people classified as female sex assigned at birth but who do not identify as women. We recognize that transgender men and other gender-diverse people may experience unique barriers to $\mathrm{HCV}$ screening and linkage to $\mathrm{HCV}$ care. Future work should focus on the specific HCV care needs of this key population. Though we validated Onomap for use in the $\mathrm{BC}$ population, it is not able to identify all people, in particular: those who would describe themselves as having a mixed ethnicity; people whose surnames are not specific to ethnic groups, and; people who adopt surnames of another ethnic group. Onomap does not identify people with Indigenous ethnicity. Due to legislated forced assimilation in Canada, many Indigenous peoples' names were changed to biblical or other European names [59]. Thus, there is a misclassification of various ethnic groups through this methodology. We used diagnostic codes in administrative datasets to assess history of mental illness and substance use. This raises several issues: bias towards underestimating prevalence in those less engaged in healthcare, and potential misclassification related to sensitivity and specificity of these measures. Potentially lower linkage rates in some key groups would result in less representation, especially people who are homeless, street-involved, and incarcerated [19].

\section{Conclusions}

This study has shown that women are progressing similarly to men across the $\mathrm{HCV}$ care cascade stages. However, gaps remain for some groups of women, particularly baby boomers and younger women, women experiencing poverty and social isolation, women with problematic substance use, and women with mental health disorders. Though access to HCV testing and treatment has expanded dramatically with DAAs, systemic barriers to testing and treatment in $\mathrm{BC}$, especially within primary care and community-based health and social services [60], disproportionately impact marginalized populations. Programming that is peer-based and specifically reaches out to support women to engage or re-engage with the HCV care cascade could help $\mathrm{BC}$ reach $\mathrm{HCV}$ elimination targets, as well as achieve equity of health care access and outcomes. Such programming must understand and address the overlapping challenges faced by women living with $\mathrm{HCV}$, as they are frequently gendered and exacerbate barriers to engaging in any form of healthcare.

\section{Supplementary Information}

The online version contains supplementary material available at https://doi. org/10.1186/s12905-021-01470-7.

Additional file 1. Supplementary Materials.

\section{Acknowledgements}

We respectfully acknowledge the traditional territories of all First Nations peoples in British Columbia, and that this work was carried out on the traditional, ancestral, and unceded territories of the Coast Salish peoples. We also gratefully acknowledge the residents of British Columbia who are represented in the BC-HTC, and for whom this work is intended to benefit. We are thankful to Emma Currie (HepC BC) and Hermione Jefferis (Aids Vancouver Island) for contributing their valuable time and insight to this research. Special thanks to the Pacific Aids Network (PAN) and the Hepatitis C Leadership Project, specifically Janet Madsen, Stacy Leblanc, and Monte Strong, for their tireless commitment to community and for facilitating our engagement and knowledge translation efforts. We acknowledge the assistance of the Provincial Health Services Authority, BC Ministry of Health, and BC Cancer staff involved in data access, procurement, and management. Thank you so the entire BC Hepatitis Testers Cohort study team.

\section{Disclaimer}

All inferences, opinions, and conclusions drawn in this manuscript are those of the authors, and do not reflect the opinions or policies of the Data Steward(s).

\section{Authors' contributions}

MEP conceived of the study, interpreted the data, and wrote the initial draft of the work. SRB contributed to the study conception, interpretation of data, and substantively revised the work. AY contributed to the study design, data analysis and interpretation. JL, CR, MB, HVG DJ, EC, PA, HS, EY, CE, LS, NP and JW contributed to the interpretation of the data and substantively revised the draft. SW contributed to the study design, data analysis and interpretation. MA contributed to the study design, data acquisition, and substantively revised the draft. MK and NJ contributed to the study conception and design, data acquisition and analysis, interpretation, and substantively revised the draft. All authors have approved the submitted version of the manuscript and have agreed to be personally accountable to their contributions and to ensure that questions related to the accuracy or integrity of any part of the work, even ones in which the author was not personally involved, are appropriately investigated, resolved, and the resolution documented in the literature. All authors read and approved the final manuscript.

\section{Funding}

M.E.P. was supported by a Canadian Institutes of Health Research Health Systems Impact Fellowship, which had no role in the study design, data collection, analysis, interpretation, or in writing the manuscript.

\section{Availability of data and materials}

All data generated or analysed during this study are included in this published article [and its supplementary information files]. 


\section{Declarations}

\section{Ethics approval and consent to participate}

BC-HTC data are de-identified and analyzed anonymously; thus, informed consent was not required. Institutional ethics approval was provided by the University of British Columbia Research Ethics Board (H14-01649) and all research was carried out in accordance with relevant guidelines and regulations.

\section{Consent for publication}

Not required.

\section{Competing interests}

SRB has received speakers' honoraria and participated in medical advisory board programs with Gilead Science (all personal payments given as unrestricted donation to BCCDC Foundation). All other authors declare no conflicts of interest.

\section{Author details}

${ }^{1}$ British Columbia Centre for Disease Control, Vancouver, BC, Canada. ${ }^{2}$ School of Population and Public Health, University of British Columbia (UBC), Vancouver, BC, Canada. ${ }^{3}$ Department of Pathology and Laboratory Medicine, UBC, Vancouver, BC, Canada. ${ }^{4}$ AIDS Network Kootenay Outreach and Support Society, Kimberly, BC, Canada. ${ }^{5}$ East Kootenay Network of People who Use Drugs, Kimberly, BC, Canada. ${ }^{6}$ British Columbia Hepatitis Network Society, Vancouver, BC, Canada. 'Division of Gastroenterology, Department of Medicine, UBC, Vancouver, BC, Canada. ${ }^{8}$ Vancouver General Hospital, Vancouver, BC, Canada. ${ }^{9}$ Department of Obstetrics and Gynecology, UBC, Vancouver, Canada. ${ }^{10} \mathrm{BC}$ Women's Hospital Research Institute, Vancouver, BC, Canada. ${ }^{11}$ Division of Infectious Diseases, Department of Pediatrics, UBC, Vancouver, Canada. ${ }^{12}$ BC Children's Hospital Research Institute, Vancouver, BC, Canada. ${ }^{13}$ Division of Infectious Diseases, Department of Medicine, UBC, Vancouver, BC, Canada.

Received: 1 April 2021 Accepted: 31 August 2021

Published online: 13 September 2021

\section{References}

1. Baden R, Rockstroh JK, Buti M. Natural history and management of hepatitis C: does sex play a role? J Infect Dis. 2014;209 Suppl 3:S81-5.

2. Corsi DJ, Karges W, Thavorn K, Crawley AM, Cooper CL. Influence of female sex on hepatitis $C$ virus infection progression and treatment outcomes. Eur J Gastroenterol Hepatol. 2016;28(4):405-11.

3. Kushner T, Terrault NA. Hepatitis C in pregnancy: a unique opportunity to improve the hepatitis $C$ cascade of care. Hepatol Commun. 2019:3(1):20-8.

4. Ropponen A, Sund R, Riikonen S, Ylikorkala O, Aittomaki K. Intrahepatic cholestasis of pregnancy as an indicator of liver and biliary diseases: a population-based study. Hepatology. 2006;43(4):723-8.

5. Safir A, Levy A, Sikuler E, Sheiner E. Maternal hepatitis B virus or hepatitis $C$ virus carrier status as an independent risk factor for adverse perinatal outcome. Liver Int Off J Int Assoc Study Liver. 2010;30(5):765-70.

6. Boucher M, Gruslin A. No. 96-The reproductive care of women living with hepatitis C infection. J Obstet Gynaecol Can. 2017;39(7):e1-e25.

7. Benova L, Mohamoud YA, Calvert C, Abu-Raddad LJ. Vertical transmission of hepatitis $C$ virus: systematic review and meta-analysis. Clin Infect Dis. 2014:59(6):765-73.

8. Esmaeili A, Mirzazadeh A, Morris MD, Hajarizadeh B, Sacks HS, Maher L, et al. The effect of female sex on hepatitis $C$ incidence among people who inject drugs: results from the International Multicohort InC3 Collaborative. Clin Infect Dis. 2018;66(1):20-8.

9. Patrick SW, Bauer AM, Warren MD, Jones TF, Wester C. Hepatitis C virus infection among women giving birth - Tennessee and United States, 2009-2014. MMWR Morb Mortal Wkly Rep. 2017;66(18):470-3.

10. Socias ME, Shannon K, Montaner JS, Guillemi S, Dobrer S, Nguyen P, et al. Gaps in the hepatitis C continuum of care among sex workers in Vancouver, British Columbia: implications for voluntary hepatitis $C$ virus testing, treatment and care. Can J Gastroenterol Hepatol. 2015;29(8):411-6.

11. Swan D, Long J, Carr O, Flanagan J, Irish H, Keating S, et al. Barriers to and facilitators of hepatitis $C$ testing, management, and treatment among current and former injecting drug users: a qualitative exploration. AIDS patient care and STDs. 2010;24(12):753-62.

12. Staton M, Ciciurkaite G, Havens J, Tillson M, Leukefeld C, Webster M, et al. Correlates of injection drug use among rural appalachian women. J Rural Health. 2018;34(1):31-41.

13. Falade-Nwulia O, Suarez-Cuervo C, Nelson DR, Fried MW, Segal JB, Sulkowski MS. Oral direct-acting agent therapy for hepatitis $C$ virus infection: a systematic review. Ann Intern Med. 2017;166(9):637-48.

14. The Canadian Network on Hepatitis C Blueprint Writing Committee and Working Groups. Blueprint to Inform Hepatitis C Elimination Efforts in Canada. Montreal, QC: CanHepC; 2019.

15. Bartlett SR, Yu A, Chapinal N, Rossi C, Butt Z, Wong S, et al. The population level care cascade for hepatitis C in British Columbia, Canada as of 2018 . impact of direct acting antivirals. Liver Int Off J Int Assoc Study Liver. 2019;39(12):2261-72.

16. Sokol R, Fisher E. Peer support for the hardly reached: a systematic review. Am J Public Health. 2016;106(7):e1-8.

17. Marshall AD, Saeed S, Barrett L, Cooper CL, Treloar C, Bruneau J, et al. Restrictions for reimbursement of direct-acting antiviral treatment for hepatitis C virus infection in Canada: a descriptive study. CMAJ open. 2016;4(4):E605-E14

18. British Columbia Ministry of Health. BC Pharmacare Newsletter. In: Division MBaPS, editor. 2017.

19. Janjua NZ, Kuo M, Chong M, Yu A, Alvarez M, Cook D, et al. Assessing hepatitis $C$ Burden and Treatment Effectiveness through the British Columbia Hepatitis Testers Cohort (BC-HTC): Design and Characteristics of Linked and Unlinked Participants. PLoS One. 2016:11(3):e0150176.

20. Islam N, Krajden M, Gilbert M, Gustafson P, Yu A, Kuo M, et al. Role of primary T-cell immunodeficiency and hepatitis B coinfection on spontaneous clearance of hepatitis C: The BC Hepatitis Testers Cohort. J Viral Hepat. 2017:24(5):421-9.

21. Trubnikov M, Yan P, Archibald C. Estimated prevalence of Hepatitis C Virus infection in Canada, 2011. Can Commun Dis Rep. 2014;40(19):429-36.

22. Pampalon R, Hamel D, Gamache P, Philibert MD, Raymond G, Simpson A. An area-based material and social deprivation index for public health in Quebec and Canada. Can J Public Health. 2012;103(8 Suppl 2):S17-22.

23. Lakha F, Gorman DR, Mateos P. Name analysis to classify populations by ethnicity in public health: validation of Onomap in Scotland. Public Health. 2011;125(10):688-96.

24. Mateos P. A review of name-based ethnicity classification methods and their potential in population studies. Population, Space and Place. 2007:13(4):243-63.

25. Mateos P, Longley PA, O'Sullivan D. Ethnicity and Population Structure in Personal Naming Networks. 2011;6(9):e22943.

26. Smith L, Norman P, Kapetanstrataki M, Fleming S, Fraser LK, Parslow RC, et al. Comparison of ethnic group classification using naming analysis and routinely collected data: application to cancer incidence trends in children and young people. BMJ open. 2017;7(9):e016332.

27. Currie E, Jefferis H, Lamb J, Reitz C, Westerby J, Pearce ME. Hep C in Focus: Who Are We Missing? Changing the Hep C Cascade of Care for Women [Webinar]. Pacific AIDS Network; 2020.

28. Janjua NZ, Islam N, Wong J, Yoshida EM, Ramji A, Samji H, et al. Shift in disparities in hepatitis $\mathrm{C}$ treatment from interferon to DAA era: $\mathrm{A}$ population-based cohort study. J Viral Hepat. 2017;24(8):624-30.

29. Janjua NZ, Yu A, Kuo M, Alvarez M, Cook D, Wong J, et al. Twin epidemics of new and prevalent hepatitis C infections in Canada: BC Hepatitis Testers Cohort. BMC Infect Dis. 2016:16:334.

30. Shah H, Bilodeau M, Burak KW, Cooper C, Klein M, Ramji A, et al. The management of chronic hepatitis C: 2018 guideline update from the Canadian Association for the Study of the Liver. Canadian Medical Association Journal. 2018;190(22):E677-E87

31. Castrejon M, Chew KW, Javanbakht M, Humphries R, Saab S, Klausner JD. Implementation of a Large System-Wide Hepatitis C Virus Screening and Linkage to Care Program for Baby Boomers. Open Forum Infect Dis. 2017;4(3): ofx109.

32. Altekruse SF, Henley SJ, Cucinelli JE, McGlynn KA. Changing hepatocellular carcinoma incidence and liver cancer mortality rates in the United States. Am J Gastroenterol. 2014;109(4):542-53.

33. Yu JW, Sun $L J$, Zhao YH, Kang P, Yan BZ. Impact of sex on virologic response rates in genotype 1 chronic hepatitis $C$ patients with peginterferon alpha-2a and ribavirin treatment. Int J Infect Dis. 2011;15(11):e740-6. 
34. Pearce M, Yu A, Alvarez M, Bartlett SR, Binka M, Jeong D, et al., editors. Frequency of Prenatal Hepatitis C Screening and Subsequent Diagnoses in British Columbia, 2018. Perinatal Services BC Healthy Mothers and Healthy Babies Conference; 2020 February 21-22, 2020; Vancouver, BC.

35. Kanwal F, Kramer J, El-Serag H, Frayne S, Cao Y, Taylor T, et al. Race and Gender Differences in the Use of Direct Acting Antiviral Agents for Hepatitis C Virus. Clinical Infectious Diseases. 2016;63(3):291-9.

36. Gowda C, Kennedy S, Glover C, Prasad MR, Wang L, Honegger JR. Enhanced identification of maternal hepatitis $C$ virus infection using existing public health surveillance systems. Paediatr Perinat Epidemiol. 2018;32(4):401-10.

37. Koneru A, Nelson N, Hariri S, Canary L, Sanders KJ, Maxwell JF, et al. Increased Hepatitis C Virus (HCV) Detection in Women of Childbearing Age and Potential Risk for Vertical Transmission - United States and Kentucky, 2011-2014. MMWR Morb Mortal Wkly Rep. 2016;65(28):705-10.

38. Page K, Leeman L, Bishop S, Cano S, Bakhireva LN. Hepatitis C Cascade of Care Among Pregnant Women on Opioid Agonist Pharmacotherapy Attending a Comprehensive Prenatal Program. Matern Child Health J. 2017;21(9):1778-83.

39. Gartner K, Elliott K, Smith M, Pearson H, Hunt G, Martin RE. "People in regular society don't think you can be a good mother and have a substance use problem": Participatory action research with women with substance use in pregnancy. Can Fam Physician. 2018;64(7):e309-e16.

40. Carter AJ, Bourgeois S, O'Brien N, Abelsohn K, Tharao W, Greene S, et al. Women-specific HIV/AIDS services: identifying and defining the components of holistic service delivery for women living with HIV/AIDS. J Int AIDS Soc. 2013;16:17433.

41. Gross MS, Ruth AR, Rasmussen SA. Respect women, promote health and reduce stigma: ethical arguments for universal hepatitis $C$ screening in pregnancy. J Med Ethics. 2020.

42. Greenaway C, Makarenko I, Tanveer F, Janjua N. Addressing hepatitis C in the foreign-born population: $A$ key to hepatitis $C$ virus elimination in Canada. Canadian Liver Journal. 2018;1(2):34-50.

43. Pearce ME, Jongbloed K, Demerais L, MacDonald H, Christian WM, Sharma R, et al. "Another thing to live for": Supporting HCV treatment and cure among Indigenous people impacted by substance use in Canadian cities. Int J Drug Policy. 2019;74:52-61.

44. Kim NJ, Locke CJ, Park H, Magee C, Bacchetti P, Khalili M. Race and Hepatitis C Care Continuum in an Underserved Birth Cohort. Journal of general internal medicine. 2018.

45. Shearer Lambert J, McHugh T, Perry N, Murphy L, Walsh J, Mantoy-Meade $P$, et al. Development of hepfriend; a dublin community hepatitis $\mathrm{C}$ peer support model. International Journal of Integrated Care. 2018;18.

46. Ko JY, Haight SC, Schillie SF, Bohm MK, PM. D. National Trends in Hepatitis CInfection by Opioid Use Disorder Status Among Pregnant Women at Delivery Hospitalization - United States, 2000-2015. Morbidity and Mortality Weekly Report. 2019;68(39):833-8.

47. Bartlett SR, Yu A, Wong S, Pearce ME, Alvarez M, Butt A, et al., editors. Current opioid agonist therapy is associated with hepatitis $C$ virus treatment uptake among people who inject drugs in a population-based data linkage study. Canadian Liver Meeting; 2020 Feb 28-Mar 1; Montreal.
48. Bartlett SR, Yu A, Wong S, Pearce ME, Alvarez M, Butt A, et al., editors. Continued Inequities In The Hepatitis C Cascade Of Care Experienced By People Who Inject Drugs In British Columbia In 2019: A Population-Level Linked Data Study. American Association for the Study of Liver Disease (AASLD); 2020 Nov 13-16; ONLINE.

49. Pedersen JS, Dong H, Small W, Wood E, Nguyen P, Kerr T, et al. Declining trends in the rates of assisted injecting: a prospective cohort study. Harm Reduct J. 2016;13:2.

50. Collins AB, Boyd J, Cooper HLF, McNeil R. The intersectional risk environment of people who use drugs. Soc Sci Med. 2019;234:112384.

51. Miller CL, Spittal PM, LaLiberte N, Li K, Tyndall MW, O'Shaughnessy MV, et al. Females experiencing sexual and drug vulnerabilities are at elevated risk for HIV infection among youth who use injection drugs. J Acq Immun Def Synd. 2002;30(3):335-41.

52. Statistics Canada. Canadian Community Health Survey - Mental Health (CCHS). 2012. Contract No.: Tables 13-10-0465-01 Mental health indicators.

53. Statistics Canada. Mental health characteristics: Perceived need for mental health care. 2018. Contract No.: Tables 13-10-0619-01 Mental health characteristics: Perceived need for mental health care.

54. Ho SB, Brau N, Cheung R, Liu L, Sanchez C, Sklar M, et al. Integrated Care Increases Treatment and Improves Outcomes of Patients With Chronic Hepatitis C Virus Infection and Psychiatric Illness or Substance Abuse. Clin Gastroenterol Hepatol. 2015;13(11):2005-14.e1-3.

55. Kestler M, Murray M, Money D, Sauve L, Pick N. The Oak Tree Clinic: The Envisioned Model of Care for Women Living with Human Immunodeficiency Virus in Canada. Women's health issues: official publication of the Jacobs Institute of Women's Health. 2018;28(2):197-8.

56. Wong WWL, Tu HA, Feld JJ, Wong T, Krahn M. Cost-effectiveness of screening for hepatitis C in Canada. CMAJ. 2015;187(3):E1 10-E21.

57. Krajden M, Cook DA, Wong $S$, Yu A, Butt ZA, Rossi $C$, et al. What is killing people with hepatitis $C$ virus infection? Analysis of a population-based cohort in Canada. Int J Drug Policy. 2019;72:114-22.

58. Hamadeh A, Haines A, Feng Z, Thein HH, Janjua NZ, Krahn M, et al. Estimating chronic hepatitis $C$ prevalence in British Columbia and Ontario, Canada, using population-based cohort studies. J Viral Hepat. 2020;27(12):1419-29.

59. Joseph B. 21 Things You May Not Know About the Indian Act: Helping Canadians Make Reconciliation With Indigenous Peoples a Reality. Port Coquitlam: Indigenous Relations Press; 2018. 189 p.

60. British Columbia Centre for Disease Control. BCCDC HCV Stakeholder Survey Highlights. 2020. Available at: https://hepatitiseducation.med.ubc. ca/providers/. Accessed July 24, 2021.

\section{Publisher's Note}

Springer Nature remains neutral with regard to jurisdictional claims in published maps and institutional affiliations.

\footnotetext{
Ready to submit your research? Choose BMC and benefit from:

- fast, convenient online submission

- thorough peer review by experienced researchers in your field

- rapid publication on acceptance

- support for research data, including large and complex data types

- gold Open Access which fosters wider collaboration and increased citations

- maximum visibility for your research: over $100 \mathrm{M}$ website views per year
}

At BMC, research is always in progress.

Learn more biomedcentral.com/submissions 